Rabaska

Revue d'ethnologie de l'Amérique française

\title{
Chaire de recherche McCain en ethnologie acadienne (Université de Moncton)
}

\section{Ronald Labelle}

Volume 10, 2012

URI : https://id.erudit.org/iderudit/1013578ar

DOI : https://doi.org/10.7202/1013578ar

Aller au sommaire du numéro

Éditeur(s)

Société québécoise d'ethnologie

ISSN

1703-7433 (imprimé)

1916-7350 (numérique)

Découvrir la revue

Citer ce document

Labelle, R. (2012). Chaire de recherche McCain en ethnologie acadienne

(Université de Moncton). Rabaska, 10, 310-313.

https://doi.org/10.7202/1013578ar d'utilisation que vous pouvez consulter en ligne. 
Conrad Laforte (p. 19-47) ; et « Bibliographie de Conrad Laforte » (p. 323-329). L'ouvrage a été lancé le 12 novembre 2011 au Musée des Ursulines de Québec sous les auspices de la Société québécoise d'ethnologie en présence de Madame Hélène Laforte et de sa fille Esther.

D. RAPPORT - Une vingtaine de membres du Groupe de recherche en études acadiennes (GRÉA) se sont réunis régulièrement à l'Université Sainte-Anne au cours de l'année 2011 avec l'intention d'encourager la recherche dans leur domaine de recherche commun. Leurs délibérations ont débouché sur la rédaction d'un document de réflexion qui a été officiellement remis aux autorités de l'institution le 31 janvier 2012 : Le Centre pluridisciplinaire en études acadiennes de l'Université Sainte-Anne. Proposition du Groupe de recherche en études acadiennes (GRÉA) sur l'avenir des études acadiennes à l'Université Sainte-Anne (Document de travail préparé par Jean-Pierre Pichette. Pointe-de-l'Église, Centre acadien, Université Sainte-Anne, janvier 2012, 71 p.). Ce rapport, envisagé comme un point de départ plutôt qu'un point d'arrivée, vise à orienter les décideurs dans la conduite d'une offensive commune, en étroite collaboration avec les chercheurs, afin d'intégrer la recherche aux priorités de l'institution.

\section{Autres}

On trouvera dans le portail $w w w$. sainteanne.ca/cofram quelques documents relatifs aux activités de la chaire, notamment le programme des deux volets du colloque international sur L'Apport des prêtres et religieux au patrimoine des minorités tenu en octobre (31 p.) et en novembre (29 p.), et les bulletins d'information préparés au Centre acadien : La Mouvée, bulletin d'information de la COFRAM ( $\mathrm{n}^{\circ} 7$, décembre 2011-janvier 2012, 12 p.) et le GRÉA (n ${ }^{\circ}$, hiver 2012, 16 p.).

JeAn-Pierre Pichette

\section{Chaire de recherche McCain en ethnologie acadienne}

Cremea, Département d'études françaises

Université de Moncton

Moncton (Nouveau-Brunswick)

E1A 3E9
Téléphone : (506) 858-4412

Courriel : ronald.labelle@umoncton.ca

Toile : www.umoncton.ca/crmea

\section{Recherche sur les contes}

Le projet de recherche financé par la FésR intitulé « Le conte traditionnel en Acadie - du déclin au renouveau » s'est terminé en mai 2012. Grâce à l'embauche d'une assistante qui détient un diplôme de maîtrise en français de l'Université de Moncton, un inventaire presque exhaustif des collections sonores de contes acadiens déposés en archives a été réalisé. Près de 4000 enregistrements de contes acadiens y ont été répertoriés, ce qui confirme la place importante du conte traditionnel acadien dans le patrimoine immatériel de l'humanité. Le projet jette les bases pour une éventuelle anthologie du conte populaire en Acadie. 


\section{Stage à l'Université de Poitiers}

La participation de Ronald Labelle cette année au Programme de mobilité professorale Moncton-Poitiers a permis de réaliser plusieurs objectifs. En plus de présenter trois conférences à l'Université de Poitiers, il a eu des rencontres avec des professeurs et chercheurs membres du groupe de recherche Mіммос (Mémoire, identités, marginalités dans le monde occidental contemporain), ce qui augure bien pour des collaborations futures. Il a aussi profité de son passage à Poitiers pour effectuer des recherches dans le fonds Geneviève Massignon à la Bibliothèque nationale de France. Des démarches ont été entreprises en vue d'obtenir des copies des contes et chansons recueillis en Acadie par Massignon en 1946 et en 1961, en vue de futures projets de publication.

\section{Projet de colloque}

Au cours de l'hiver 2012, la chaire CREMEA a mis sur pied un comité organisateur d'un colloque international sur la chanson et la musique acadiennes. Ce comité comprend, entre autres, des représentants du département de musique de l'Université de Moncton et du Centre d'études acadiennes Anselme-Chiasson. Des démarches seront entreprises par le comité afin d'obtenir les fonds nécessaires à la venue de chercheurs en musique traditionnelle de la Louisiane et de l'Ouest de la France. Un des buts du colloque sera d'étudier les façons dont la musique traditionnelle a été réinterprétée et transformée pour donner lieu à de nouvelles formes musicales en Acadie et en Louisiane.

\section{Projet d'édition critique}

En février 2012, nous avons complété une première version de l'édition critique des Contes d'Acadie de Thomas LeBlanc. Le texte a alors été remis au comité éditorial du Groupe de recherche en édition critique.

\section{Articles et chapitres de livres publiés}

- " J.-Thomas LeBlanc et le mystère des Contes d'Acadie », dans Port-Acadie, $\mathrm{n}^{\text {os }}$ 20-21, 2011-2012, p. 131-139.

- «L'Établissement et la transmission du répertoire traditionnel : le chanteur Allain Kelly », dans " M'amie, faites-moi un bouquet », Mélanges posthumes autour de l'œuvre de Conrad Laforte, sous la direction de J.-N. De Surmont et S. Gauthier, Québec, Presses de l'Université Laval, «Archives de folklore» 30, 2011, p. 255-272. - «Les Archives sonores dans les provinces de l'Atlantique - un patrimoine en perdition? », dans Archival Narratives for Canada-Re-Telling Stories in a Changing Landscape, Kathleen Garay \& Christl Verduyn (dir.), Halifax \& Winnipeg, Fernwood Publishing, 2011, p. 87-97.

- "Une "Amérique française" plutôt québécoise où le patrimoine immatériel n’échappe pas au matériel », dans Rabaska, vol. 9, 2011, p. 230-235.

- «Les Tailleurs de pierre de Shédiac et la construction de la cathédrale » dans La Cathédrale Notre-Dame-de-l'Assomption : Monument de la Reconnaissance, par Robert Pichette, Moncton, Chaire de recherche en études acadiennes, 2012, p. 255-257. - « Oudjigeaskwa, Cendrillon Mi'kmaq », dans le collectif Cendrillon sur quatre continents, Moncton, Bouton d'or Acadie, 2011, p. 85-96. 
Conférences prononcées par le titulaire

- « 100 ans de recherches sur les traditions orales en Acadie », Département de musicologie, Université de Poitiers, mars 2012.

- «La Chanson traditionnelle canadienne-française illustrée », conférence publique, Université de Poitiers, mars 2012.

- « De l'Ouest de la France à l'Est du Canada : le parcours ethnographique de Geneviève Massignon ", Journées scientifiques internationales sur le thème des interactions patrimoniales franco-québécoises, Faculté des sciences humaines et arts, Université de Poitiers, mars 2012.

- Conférence d'ouverture, «L'Apport des prêtres et religieux au patrimoine des minorités - parcours comparés Bretagne / Canada français », Journées internationales d'étude, Université Sainte-Anne, Nouvelle-Écosse, octobre 2011.

- «Catherine Jolicœur, Fille de Marie de l'Assomption », L'Apport des prêtres et religieux au patrimoine des minorités - parcours comparés Bretagne / Canada français, Université Sainte-Anne, Pointe-de-l'Église, Nouvelle-Écosse, octobre 2011, et Université de Bretagne Occidentale, Brest, France, novembre 2011.

- «Acadian Vocal and Instrumental Traditions », conférence publique, Celtic Colours Folk Festival, Cape Breton University, Centre for Cape Breton Studies, Sydney, N.-É., octobre 2011.

\section{Activités de diffusion}

- Suite au projet complété en 2010 intitulé « Mise en valeur des ressources culturelles franco-terreneuviennes ", les responsables des services de langue française de la province de Terre-Neuve-et-Labrador ont accepté de financer la numérisation et l'indexation de tout le fonds du Centre d'études franco-terreneuviennes, conservé à l'Université Memorial. Le titulaire a été consulté, à l'automne 2011, en vue de la mise en œuvre de cet important projet patrimonial.

- L'exposition intitulée «L'Art du conte en Acadie », organisée conjointement par la Chaire de recherche McCain, le Musée acadien et le Centre d'études acadiennes, a pris fin en octobre 2011. La chaire CREMEA a participé à l'organisation des deux dernières soirées de contes qui ont accompagné l'exposition en septembre et en octobre. - Dans le cadre du $4^{\mathrm{e}}$ forum des sociétés historiques acadiennes tenu à Grand-Pré, N.-É., en octobre 2011, le titulaire a animé une table ronde sur l'utilisation de la chanson traditionnelle dans les musées, les sites historiques et les écoles de langue française.

- Le film intitulé La Tapisserie du French Shore / Phantoms of the French Shore, dont le titulaire avait participé au tournage en novembre 2010, a été diffusé sur les ondes de Radio-Canada en avril 2012.

\section{Réflexions du titulaire au moment de son départ}

Ce rapport marque la fin de nos activités en tant que titulaire de la Chaire de recherche McCain en ethnologie acadienne. En quittant l'Université de Moncton pour occuper un poste à Cape Breton University, nous sommes fiers d'avoir contribué à faire rayonner l'ethnologie acadienne dans le milieu universitaire au cours des six dernières années. Nous espérons que l'ethnologie acadienne conservera une place 
à l'Université de Moncton, car les besoins sont grands, autant au niveau de l'enseignement au sein de la Faculté des arts et des sciences sociales qu'au niveau de la recherche et de la conservation au Centre d'études acadiennes Anselme-Chiasson.

RonALD LABELLE

\section{Centre d'études acadiennes Anselme-Chiasson}

Université de Moncton

Moncton (N.-B.) E1A 3E9
Téléphone : (506) 858-4085

Télécopieur : (506) 858-4530

Courriel : robert.richard@umoncton.ca

Toile : www.umoncton.ca/umcm-ceaac

\section{Laboratoire de conservation}

Dans les 320 heures octroyées par une subvention obtenue du Programme national de développement des archives (PNDA) 2011-2012, les chargés de projet Léandre Bourgeois et Daniel Fournier, de pair avec le soutien de Robert Richard, ont converti en signal numérique 1388 supports magnétiques additionnels. Malheureusement, avec l'abolition du PNDA par Bibliothèque et Archives Canada (30 avril 2012), l'espoir de succès de la deuxième demande soumise s'est éteint. Néanmoins, le Centre d'études acadiennes Anselme-Chiasson (CÉAAC) bénéficie actuellement, pour une durée de 14 semaines, d'un cinquième projet d'emploi du programme Jeunesse Canada au travail dans les établissements du patrimoine du ministère du Patrimoine canadien. L'étudiante embauchée, Véronique Blanchard, a amorcé la migration du signal analogique-numérique ainsi que le marquage numérique des 900 bobines audio restantes à numériser aux archives de folklore du CÉAAC. En outre, elle a déjà numérisé 305 des 572 bobines analogiques de la collection sonore Catherine-Jolicœur.

\section{Acquisitions}

Le collecteur Laurent Comeau a récemment complété et déposé un inventaire de cinq vidéos (noir et blanc) d'une séance tournée vers 1976 auprès de deux informateurs acadiens du Nouveau-Brunswick, Loderie St-Cœur de Fair Isle et Irénée Allain de Notre-Dame-de-Kent. Le premier interprète plusieurs reels à bouche ainsi que des pièces traditionnelles à la bombarde et à la musique à bouche ; le second fait de même avec son violon, tout en y ajoutant diverses chansons locales. Encore sur le terrain du Nouveau-Brunswick, l'automne dernier, Robert Richard s'est joint à Ronald Labelle pour deux missions de collectage, l'une avec l'informatrice de Rogersville, Flora Thibodeau née en mars 1901, et l'autre avec le violoneux acadien Eddie Poirier. L'archiviste en ethnologie acadienne a poursuivi ses entrevues avec les nonagénaires Dora et Angeline Landry de l'Anse-des-Cormier (quartier de Memramcook) et le centenaire François Bourque de Cocagne. 